\title{
Do All Supernovae Beget Pulsars?
}

IT is odd that the Crab Nebula is still the only supernova remnant definitely associated with a pulsar. Or is it? This question will be pondered by many astronomers following the announcement this week of another possible association-but, like several other suggested associations, not definite-between a pulsar and a nearby supernova remnant. On page 229 of this issue of Nature, Davies, Lyne and Seiradakis report the detection of a pulsar about $0.6^{\circ}$ from the centre of a supernova remnant which itself is about $0.7^{\circ}$ across. In addition to the positional propinquity, the age of the pulsar estimated from the rate of rundown of the pulsations is not a bad match to the age of the supernova remnant as estimated from its appearance.

This must now be added to the possibility of an association between a patch of radio emission four or five degrees across in the southern hemisphere constellation Vela, widely suspected to be a supernova remnant, and a pulsar situated about one degree from the centre (Large, Vaughan and Mills, Nature, 220, 340 ; 1968). Another possible association, between a supernova remnant in Crux and the pulsar PSR 1154-62, is expected to be published soon.

So, of the scores of pulsars now known, the number of links with supernova remnants boils down to one definite and three possibles. Yet almost since the discovery of pulsars it has been a credendum that pulsars are formed phoenix-like in the ashes of stars which have otherwise totally disintegrated in supernova explosions. Why, then, are there not more definite associations between pulsars and supernova remnants, and do all supernovae indeed lead to the formation of pulsars?

Professor A. Hewish of the Cambridge radio astronomy group addressed himself to the latter question at last week's meeting in London of the Royal Astronomical Society, and described his answer as "a qualified yes". Professor Hewish's chief approach was a statistical one, from which he arrived at a roughly one-to-one relation between the number of pulsars in the neighbourhood of the Earth and the number of supernova remnants having the same range of ages.

To begin with, it is necessary to decide how far back in time the search must be taken. The ages of pulsars can be estimated from the ratio of the pulse period $(P)$ to the rate at which the period is lengthening $(\dot{P})$, although here it is, of course, necessary to assume a model for the nature of pulsars. Professor Hewish said that the ages of twenty-seven pulsars for which the rate of run-down can be measured are fairly tightly clustered around a mean of $5 \times 10^{6}$ years - three pulsars are younger than $10^{6}$ years and three are older than $5 \times 10^{7}$ years. Pulsars older than about $10^{8}$ years are believed to be too weak to be detected.

Pulsar distances are estimated from the degree to which the lower frequencies in the signals are delayed by electrons in the interstellar medium (which requires an assumption to be made about the interstellar electron density) and, occasionally, by examining the $21 \mathrm{~cm}$ emis- sion from pulsars for signs of absorption by intervening spiral arms of the galaxy whose distances are known. Professor Hewish said that of a sample of sixty pulsars, fifty are less than 2 kiloparsecs distant (6,500 light years), ten are at more than $2 \mathrm{kpc}$ and one is farther than $4 \mathrm{kpc}$.

The most critical assumption comes in estimating what proportion of those pulsars that are near enough to the Earth to give a signal are not detected because the lighthouse-like beam of radiation does not sweep across the Earth. Much depends on the unknown mechanism generating the extremely narrow beams of radiation, but Professor Hewish estimated that the space density of observed pulsars should be multiplied by a factor of between three and ten, with three the preferred value.

The number of nearby pulsars estimated on this basis is said by Professor Hewish to be near enough to the estimated number of supernovae younger than $5 \times 10^{6}$ years to support the view that each supernova leads to the formation of a pulsar. So why are there not more cases of pulsars being identified in the midst of supernovae remnants? Part of the story is that several of the well known remnants that have been attributed to historical observations of supernovae are too distant for any associated pulsar to be detectable. But from the evidence so far it also looks as if pulsars are formed with velocities sufficient to carry them away from the remainder of the residuum of the explosion so that it becomes difficult confidently to link a pulsar with a nearby remnant. Observations of the effects of interstellar scintillations on pulsar signals, for example, indicate pulsar transverse velocities up to several hundred kilometres a second, so that a pulsar will be about $100 \mathrm{pc}$ from its birthplace after $10^{6}$ years. Velocities of this order fit in nicely with the observation that the scale height above the plane of the galaxy is about $140 \mathrm{pc}$ for pulsars, but only $60 \mathrm{pc}$ for the $\mathrm{OB}$ stars which are thought to be the precursors of supernovae. Whether the velocities originate in a nonsymmetric supernova explosion, or whether they imply that the parent star was originally a member of a binary system of which the companion either disintegrated or was blown away, remains to be elucidated.

It is clear that not everybody will share Professor Hewish's confidence in a one-to-one correspondence between pulsars and supernova remnants - the uncertainties are such that a subclass of supernovae leading to pulsars still seems not to have been ruled out-but whatever its validity the three tentative associations so far recognized seem to fall into a pattern which suggests that those supernova remnants which were formed with a pulsar are profoundly affected by the pulsar. It is well known from Professor Gold's calculations that it is energy supplied by the Crab pulsar that keeps the Crab nebula glowing. But the pulsar seems still to be an influence on the nebula as the pulsar moves away-both the Vela remnant and the Crux remnant are brighter on the side nearest the associated pulsar.-By our Astronomy Correspondent. 